TCF7L2 variants in African American patients with bipolar disorder and high BMI

Linsey Jackson ${ }^{1}$, Brandon Coombes, PhD ${ }^{1}$, Joyce E. Balls-Berry, $\mathrm{PhD}, \mathrm{MPE}^{1}$, and Joanna Biernacka, $\mathrm{PhD}^{1}$

${ }^{1}$ Mayo Clinic

OBJECTIVES/GOALS: In a previous genome-wide association study of European Americans, rs12772424, a variant in the gene encoding transcription factor 7-like 2 (TCF7L2), was shown to have a SNP$\mathrm{BMI}$ interaction association with bipolar disorder (BD). The objective of this study was to replicate this finding in an African American (AA) sample. METHODS/STUDY POPULATION: Using a sample of 659 controls and $323 \mathrm{BD}$ cases from the Genetic Association Information (GAIN) Study of BD, we conducted analyses to assess association between $\mathrm{BD}$ and the interaction of $\mathrm{BMI}$ with genetic variation in TCF7L2. For this study we identified 4572 single nucleotide polymorphisms (SNPs) in a $1 \mathrm{Mb}$ region around the candidate SNP rs1272424. Based on variants identified in the prior analysis of the larger European American dataset we selected SNPs for analysis in the AA data. This allowed for a smaller AA sample, and still maintained adequate power for statistical significance. RESULTS/ANTICIPATED RESULTS: We anticipate observing an effect of TCF7L2 variation on the relationship between $\mathrm{BD}$ and $\mathrm{BMI}$ in the AA data. We also anticipate that combining results from the European and African American patients may help narrow down potentially functional variants in TCF7L2 that influence the association between BD and BMI. DISCUSSION/SIGNIFICANCE OF IMPACT: Psychiatric genetics studies lack ancestral diversity among participants, decreasing their generalizability, and possibly increasing health disparities, especially for diseases like $\mathrm{BD}$, which is often misdiagnosed and untreated in AAs. This work propels us towards understanding the genetics of $\mathrm{BD}$ and obesity in this underrepresented population.

4182

Use of Machine Learning for Surgical Outcomes Research: what are the clinical implications?

Priscila Rodrigues Armijo ${ }^{1}$, Sindhura Bonthu ${ }^{2}$, Alicia Schiller ${ }^{3}$, Qiuming Zhu ${ }^{2}$, and Tiffany Tanner ${ }^{3}$

${ }^{1}$ University of Nebraska Medical Center - Great Plains IDeA-CTR;

${ }^{2}$ University of Nebraska - Omaha; ${ }^{3}$ University of Nebraska Medical Center

OBJECTIVES/GOALS: Multivariate regression is used for surgical outcomes analyses; but does not allow for evaluation of all variables. Machine learning could be the perfect alternative to address this issue. Our aim was to evaluate whether machine learning is a feasible alternative to evaluate surgical outcomes. METHODS/STUDY POPULATION: H-CUP National Inpatient Sample database was queried for adult patients with colorectal cancer who underwent colorectal resection, while the NSQIP database was queried for adult patients with rectal cancer who underwent proctectomy. A multivariate regression analysis was performed to assess risk factors associated with 30-day complications following those procedures. Subsequently, machine learning techniques of under-sampling and oversampling were applied to the same datasets for the evaluation of risk factors for the same outcome. These techniques were used to achieve a larger population sample size and to detect statistical significance. Results between the two methodologies were compared. RESULTS/ANTICIPATED RESULTS: Multivariate regression revealed that open approach, gender, race, geographic location, number of comorbidities, and type of insurance was associated with increased 30-day mortality in colorectal resection patients. Conversely, the use of machine learning revealed that preoperative weight loss, preexistent chest heart failure, renal failure or perivascular disease were strongly associated with 30 -day mortality. For proctectomy patients, multivariate regression found no association between surgical approach and 30-day mortality. However, machine learning revealed gender, hypertension, and reoperation to be strongly associated with 30-day mortality. DISCUSSION/ SIGNIFICANCE OF IMPACT: Machine learning enabled multiple combinations that were not possible to examine in a conventional multivariate regression analysis. Machine learning compared to traditional multivariate regression produced significantly different outcomes, highlighting the need for in depth of these methodologies.

4179

\section{Use of tobacco products and their association with wheezing among adult current tobacco users in the US - Findings from The Population Assessment of Tobacco and Health Study (2015-2016)*}

Liane M Schneller ${ }^{1}$, Zahíra Quiñones-Tavárez, Maciej Goniewicz, Zidian Xie, Scott McIntosh, Richard O'Connor, Deborah J. Ossip, $\mathrm{PhD}^{1}$, Irfan Rahman, and Dongmei Li

${ }^{1}$ University of Rochester Medical Center

OBJECTIVES/GOALS: Wheezing has been shown to be associated with use of cigarettes, and more recently, electronic nicotine delivery systems (ENDS). This study assessed the association of poly use of tobacco products with wheezing among a national representative sample of US adult current tobacco users. METHODS/STUDY POPULATION: Data from the Population Assessment of Tobacco and Health (PATH) Study Wave 3 (W3) were used. Weighted prevalences of self-reported wheezing and related respiratory symptoms for non-users compared to users of cigarettes, ENDS, cigars, and any combination of these products (poly use of tobacco products) were presented for 28,082 adults. The cross-sectional association of tobacco use with self-reported wheezing and other related respiratory symptoms was assessed using weighted multivariable and ordinal logistic regression with consideration of complex sampling design. RESULTS/ANTICIPATED RESULTS: Most adults who reported on wheezing symptoms did not currently use cigarettes, ENDS or cigars (79\%), 15\% used cigarettes, 3\% used a combination of cigarettes, ENDS and cigars, $1 \%$ used ENDS, and $1 \%$ used cigars. Significantly higher odds of ever had wheezing or whistling in chest at any time in the past was observed among current cigarette (adjusted OR: 2.62, 95\%CI: 2.35, 2.91), ENDS (1.49, 95\%CI: 1.14, 1.95), and poly users $(2.67,95 \%$ CI: $2.26,3.16)$ compared to non-users. No differences were seen for cigar use. Polytobacco use was associated with a higher odds of ever wheezing when compared to ENDS $(1.61,95 \%$ CI: $1.19,2.17)$ and cigar use $(2.87,95 \% \mathrm{CI}$ : $1.93,4.26)$, but not cigarettes. DISCUSSION/ SIGNIFICANCE OF IMPACT: Wheezing is associated with the use of cigarettes, ENDS, or any combination of cigarette, ENDS and cigars likely due to the inhalation of noxious chemicals and gases found in the smoke of cigarettes and ENDS that are likely to increase the odds of experiencing wheezing. CONFLICT OF INTEREST DESCRIPTION: MLG serves as a paid consultant for Johnson \& Johnson and has received research grant from Pfizer, manufacturers of smoking cessation medications. The other authors have no conflicts to declare. 